\title{
Subclinical alveolar bleeding in pulmonary vasculitides: correlation with indices of disease activity
}

\author{
A. Schnabel*, M. Reuter**, E. Csernok*, C. Richter*, W.L. Gross*
}

Subclinical alveolar bleeding in pulmonary vasculitides: correlation with indices of disease activity. A. Schnabel, M. Reuter, E. Csernok, C. Richter, W.L. Gross. (C)ER Journals Ltd 1999.

ABSTRACT: Haemosiderin-laden alveolar macrophages are a common finding in patients with alveolar bleeding. Iron-positive macrophages, suggestive of subclinical alveolar bleeding, were found to be fairly common in bronchoalveolar lavage (BAL) fluid in primary systemic vasculitis but uncommon in collagen vascular diseases (CVDs) and rheumatoid arthritis (RA).

To substantiate the impression that subclinical alveolar bleeding may be a feature distinguishing between these disorders, fibreoptic bronchoscopy and BAL were performed in 49 patients with active Wegener's granulomatosis or Churg-Strauss syndrome and 44 patients with CVDs or RA, all of them without clinically manifest alveolar bleeding. The percentage of iron-positive cells was compared with clinical and radiological findings.

Only a minority of the CVD and RA patients had iron-positive alveolar macrophages; the 95th percentile of the median number of such cells was $5 \%$. Fifty-three per cent of the patients in the vasculitis group had $>5 \%$ iron-positive cells, with individual counts ranging up to $95 \%$. Patients with iron-positive macrophages had more extensive disease, more frequent microhaematuria, a higher antineutrophil cytoplasmic antibody titre, a higher myeloperoxidase concentration in the BAL fluid and somewhat more frequent low-attenuation opacities in pulmonary high-resolution computed tomography than the patients with a low iron-positive cell count.

In conclusion, subclinical alveolar bleeding was, indeed, a common finding in antineutrophil cytoplasmic antibody-associated vasculitis, which distinguished these disorders from lung disease due to collagen vascular diseases or rheumatoid arthritis. Its association with indices of disease activity, although weak in this cross-sectional study, merits a longitudinal study of its value for the long-term monitoring of vasculitis patients.

Eur Respir J 1999; 14: 118-124.
*Poliklinik für Rheumatologie, Medizinische Universität Lübeck and Rheumaklinik Bad Bramstedt, and $* *$ Klinik für Radiologische Diagnostik, Universität Kiel, Germany.

Correspondence: A. Schnabel

Poliklinik für Rheumatologie

Medizinische Universität Lübeck

Ratzeburger Allee 160

D-23538 Lübeck

Germany

Fax: 494515003650

Keywords: Bronchoalveolar lavage collagen vascular disease

rheumatoid arthritis

vasculitis

Wegener's granulomatosis

Received: March 91998

Accepted after revision January 271999
Alveolar bleeding due to capillaritis is a particularly severe form of immunologically mediated pulmonary small vessel disease [1]. Collections of cases suggest that systemic vasculitis, collagen vascular diseases (CVDs) and Goodpasture's syndrome caused by antiglomerular basement membrane antibodies are the most common causes of this condition in noninfected, nonhaemocytopenic patients and it was noted that, together with bone marrow transplantation, immunologically mediated vascular disease is one of the most important causes of diffuse alveolar bleeding [1-3].

Extensive alveolar bleeding due to capillaritis is a lifethreatening condition, the cardinal features of which are dyspnoea, haemoptysis, an acute drop in the haematocrit, diffuse infiltrates on chest radiographs and recovery of progressively haemorrhagic fluid on bronchoalveolar lavage (BAL) $[4,5]$. Infiltration of the alveolar septa by neutrophils, disruption of capillary vessels, diffuse interstitial bleeding and filling of alveoli with haemorrhagic material are the main histopathological findings $[1,6]$. Another common finding is haemosiderin-laden alveolar and tissue macrophages, which appear to originate from the uptake by macrophages of red cells and red cell degradation products $[1,7]$. Such cells have also been recovered by BAL in patients with overt alveolar bleeding [2, 5, 8]. Extending these findings, haemosiderin-laden alveolar macrophages were found in the BAL fluid of vasculitic patients without clinically manifest alveolar bleeding. It was also observed that such cells are fairly common in primary systemic vasculitis but uncommon in CVDs and rheumatoid arthritis (RA).

This suggested that alveolar bleeding in vasculitis may occur subclinically and that this may be a feature distinguishing primary systemic vasculitis from CVDs and RA. These questions were addressed by determining the number of iron-positive alveolar macrophages in the BAL fluid of patients with Wegener's granulomatosis (WG), ChurgStrauss syndrome, microscopic polyangiitis, systemic lupus erythematosus (SLE), polymyositis/dermatomyositis, systemic sclerosis and RA. The diagnostic significance of an 
elevated count of iron-positive macrophages was also examined by relating it to clinical findings and markers of local and systemic inflammation.

\section{Patients and methods}

\section{Patients}

Forty-nine consecutive patients with antineutrophil cytoplasmic antibody (ANCA)-associated vasculitis, 31 patients with CVD and 13 patients with RA were studied. All of these patients underwent bronchoscopy and BAL for staging of disease and evaluation of pulmonary involvement owing to the underlying condition.

The vasculitis group consisted of 23 males and 26 females, with a median age of $55 \mathrm{yrs}$ (25th-75th percentile 49-63 yrs). Forty-five of the patients in this group had WG and four had Churg-Strauss syndrome according to the 1990 American College of Rheumatology criteria [9, 10] and the 1992 Chapel Hill descriptions [11] (table 1). Twelve patients were examined at first presentation with generalized disease, the remaining 37 patients during relapse or during re-evaluation under immunosuppressive treatment. In the former group, five patients were treated with prednisolone (maximum dose $10 \mathrm{mg}$ ) and seven received no treatment; in the latter group, 22 patients were given cyclophosphamide, one received low-dose methotrexate, five cotrimoxazole, three low-dose corticosteroids and six received no treatment. All of the patients in this group underwent comprehensive evaluation, including a laboratory profile with acute phase reactants and ANCA, lung function testing, chest radiography, pulmonary high-resolution computed tomography (HRCT), BAL, otolaryngological and ophthalmological referral and cranial magnetic resonance imaging. The extent of disease was scored using the ELK system (an international scoring system for vasculitides) and the Disease Extent Index, as previously described [12]. ANCA levels were determined using a standard indirect immunofluorescence test [13]. HRCT images were assessed for septal and nonseptal lines, nodules, masses, consolidation and low-attenuation opacities and the findings evaluated according to the definitions of the Fleischer Society

Table 1. - Clinical characteristics of 49 patients with antineutrophil cytoplasm antibody (ANCA)-associated vasculitis with high $(>5 \%)$ and low $(\leq 5 \%)$ levels of iron-positive alveolar macrophages (AM) in bronchoalveolar lavage fluid

\begin{tabular}{|c|c|c|c|}
\hline & $\begin{array}{l}\text { High Fe+ } \\
\text { AM }\end{array}$ & $\begin{array}{c}\text { Low Fe+ } \\
\text { AM }\end{array}$ & $\begin{array}{c}\mathrm{p}- \\
\text { value }\end{array}$ \\
\hline Females:males $\mathrm{n}$ & $12: 14$ & $14: 9$ & \\
\hline Age yrs & $53(45-61)$ & $57(49-63)$ & \\
\hline $\begin{array}{l}\text { Duration of disease } \\
\text { months }\end{array}$ & $21(8-60)$ & $21(12-61)$ & NS \\
\hline Disease Extent Index & $6(4-7.5)$ & $4(4-5)$ & 0.021 \\
\hline $\mathrm{ESR} \mathrm{mm} \cdot \mathrm{h}^{-1}$ & $37(14-75)$ & $26(12-52)$ & NS \\
\hline $\begin{array}{l}\text { C-reactive protein } \\
\mathrm{mg} \cdot \mathrm{dL}^{-1}\end{array}$ & $0.5(0.4-2.5)$ & $0.5(0.4-1.2)$ & NS \\
\hline ANCA titre $x^{-1}$ & $256(0-256)$ & $8(0-128)$ & 0.030 \\
\hline
\end{tabular}

Data are presented median (interquartile range). ESR: erythrocyte sedimentation rate; NS: not significant.
[14]. Indications for BAL were: 1) an abnormal chest radiograph showing nodules or consolidation $(n=8)$, infiltrates $(n=11)$ or segmental atelectasis $(n=1) ; 2)$ an abnormal pulmonary HRCT showing septal or nonseptal lines $(n=21)$, nodules $(n=15)$, masses $(n=9)$, consolidation $(n=4)$ or focal ground-glass attenuation $(\mathrm{n}=9)$; or 3$)$ cough, dyspnoea or impaired lung function $(n=35)$. A final diagnosis of pulmonary involvement was made on the basis of abnormal radiological and/or bronchoscopic findings (diffuse tracheobronchitis, tracheobronchial ulceration, endobronchial granuloma or inflammatory stenoses) in 43 patients. Two patients had subclinical alveolitis in the presence of active extrapulmonary WG, with $40 \%$ lymphocytes in one patient and $17 \%$ lymphocytes plus $4 \%$ eosinophils in the other. The remaining four patients had active systemic disease but normal pulmonary findings.

The CVD group comprised 25 females and six males with a median age of 39 yrs (27-55 yrs). Five of these patients had SLE according to the 1982 American Rheumatism Association criteria [15], 14 had polymyositis or dermatomyositis according to the criteria of BOHAN and Peter [16] and 12 had systemic sclerosis according to the 1980 preliminary American Rheumatism Association criteria [17] (table 2).

The RA group consisted of nine females and four males, with a median age of 59 yrs (49-68 yrs). All of the patients in this group fulfilled the 1988 criteria of the American Rheumatism Association [18]. They were subjected to pulmonary evaluation including HRCT and BAL, because of pulmonary symptoms or abnormal chest radiography findings suggestive of interstitial lung disease.

Six of the CVD patients were examined at initial presentation without any treatment; the remaining 25 CVD patients and all of the RA patients received treatment with either low-dose methotrexate $(n=12)$, hydroxychloroquine $(n=4)$, corticosteroid monotherapy $(n=6)$, azathioprine $(n=2)$ or cyclophosphamide $(n=10)$. A final diagnosis of definitive interstitial lung disease caused by the underlying

Table 2. - Clinical characteristics and pulmonary findings in 31 patients with collagen vascular diseases and 13 patients with rheumatoid arthritis

\begin{tabular}{lc}
\hline Females:males $\mathrm{n}$ & $34: 10$ \\
Age yrs & $56(47-65)$ \\
Duration of disease months & $53(24-96)$ \\
$\mathrm{ESR} \mathrm{mm}^{-1}$ & $23(17-57)$ \\
$\mathrm{C}-\mathrm{reactive}$ protein $\mathrm{mg} \cdot \mathrm{dL}^{-1}$ & $0.8(0.4-2.1)$ \\
$\mathrm{VC} \%$ pred & $83(69-99)$ \\
$\mathrm{FEV} 1 / \mathrm{VC} \%$ & $85(77-90)$ \\
$\mathrm{DL}, \mathrm{CO} \% \mathrm{pred}$ & $84(74-91)$ \\
$\mathrm{Pa}, \mathrm{O}_{2} \mathrm{mmHg}$ & $84(77-101)$ \\
$\mathrm{Pa}, \mathrm{CO}_{2} \mathrm{mmHg}$ & $34(32-36)$ \\
$\mathrm{BAL}$ fluid cell profile & \\
Total cells $10^{3} \cdot \mathrm{mL}^{-1}$ & $224(128-342)$ \\
Lymphocytes \% & $20(10-34)$ \\
Neutrophils \% & $3(1-8)$ \\
Eosinophils \% & $1(0-2)$ \\
Macrophages \% & $72(50-81)$ \\
\hline
\end{tabular}

Data are presented as median (interquartile range). ESR: erythrocyte sedimentation rate; VC: vital capacity; FEV1: forced expiratory volume in one second; $D$ L,CO: diffusion capacity of the lung for carbon monoxide; $P \mathrm{a}, \mathrm{O}_{2}$ : arterial oxygen tension; $\mathrm{Pa}_{\mathrm{a}} \mathrm{CO}_{2}$ : arterial carbon dioxide tension; BAL: bronchoalveolar lavage. $(1 \mathrm{mmHg}=0.133 \mathrm{kPa}$. $)$ 
disease condition was made in 38 patients. In 35 patients, this diagnosis was based on increased interstitial markings on the chest radiograph or HRCT scan in association with an abnormal BAL fluid cell profile and in three patients, on increased interstitial markings in the presence of a normal BAL fluid cell profile. Six patients had subclinical alveolitis as evidenced by an abnormal BAL fluid cell pattern in the presence of normal radiological findings.

All patients were nonsmokers. Since elevated pulmonary capillary pressure can cause leakage of red cells into the terminal airways, patients with evidence of left heart failure or other reasons for increased pulmonary capillary pressure were excluded from this study. Also excluded were patients with renal insufficiency, thrombocytopenia or impaired plasmatic coagulation. No patients received intense anticoagulatory treatment, but 12 vasculitis, seven CVD and seven RA patients received prophylactic treatment with low-dose heparin. Two patients with coronary artery disease and five with peripheral artery disease received low-dose acetyl salicylate.

\section{Bronchoscopy}

Fibreoptic bronchoscopy was performed after local anaesthesia with lidocaine and premedication with atropine and a morphine antitussant. For BAL, the bronchoscope was wedged into a segmental bronchus and 10 fractions of $20 \mathrm{~mL} 0.9 \%$ sterile saline were instilled and aspirated. The recovered material was examined for conventional bacterial pathogens, acid-fast bacteria, Legionella, Chlamydia, Mycoplasma and Pneumocystis carinii and any infected material was omitted from further study. Likewise, patients with visibly haemorrhagic BAL fluid or BAL fluid containing $>10$ red cells per high-power field in the cytospin preparation were excluded from this study.

\section{Bronchoalveolar lavage analyses}

For assessment of the BAL cell profile, the pooled lavage fluid was filtered through surgical gauze and centrifuged. The cell pellet was resuspended in RPMI 1640 medium (Gibco, Eggenstein, Germany) to a density of $10^{6}$ cells $\cdot \mathrm{mL}^{-1}$ and cytospin preparations were prepared using a Shandon II cytocentrifuge (Shandon Products, Cheshire, UK) and stained using May-Grünwald Giemsa. The distribution of cell types was assessed by counting 300 cells. In the authors' laboratory, normal values are $\leq 15 \%$ lymphocytes, $\leq 5 \%$ neutrophils and $<1 \%$ eosinophils. For the enumeration of iron-positive macrophages, cytospin preparations were stained with Prussian blue (potassium ferricyanide) [19]. This chromogen combines with intracellular trivalent iron to give an intense blue colour. Airdried cytospin slides were incubated with potassium ferricyanide in 2\% hydrochloric acid for $10 \mathrm{~min}$ and subsequently counterstained using May-Grünwald Giemsa. Positive staining ranged from a faint homogeneous blue coloration of the cytoplasm to intense blue staining of bulky intracytoplasmic material. Only macrophages containing intensely staining particulate material in the cytoplasm were deemed positive (fig. 1). These cells were expressed as the percentage of total alveolar macrophages. Neither granulocytes nor lymphocytes or red cells stained positive. Previous authors have often employed
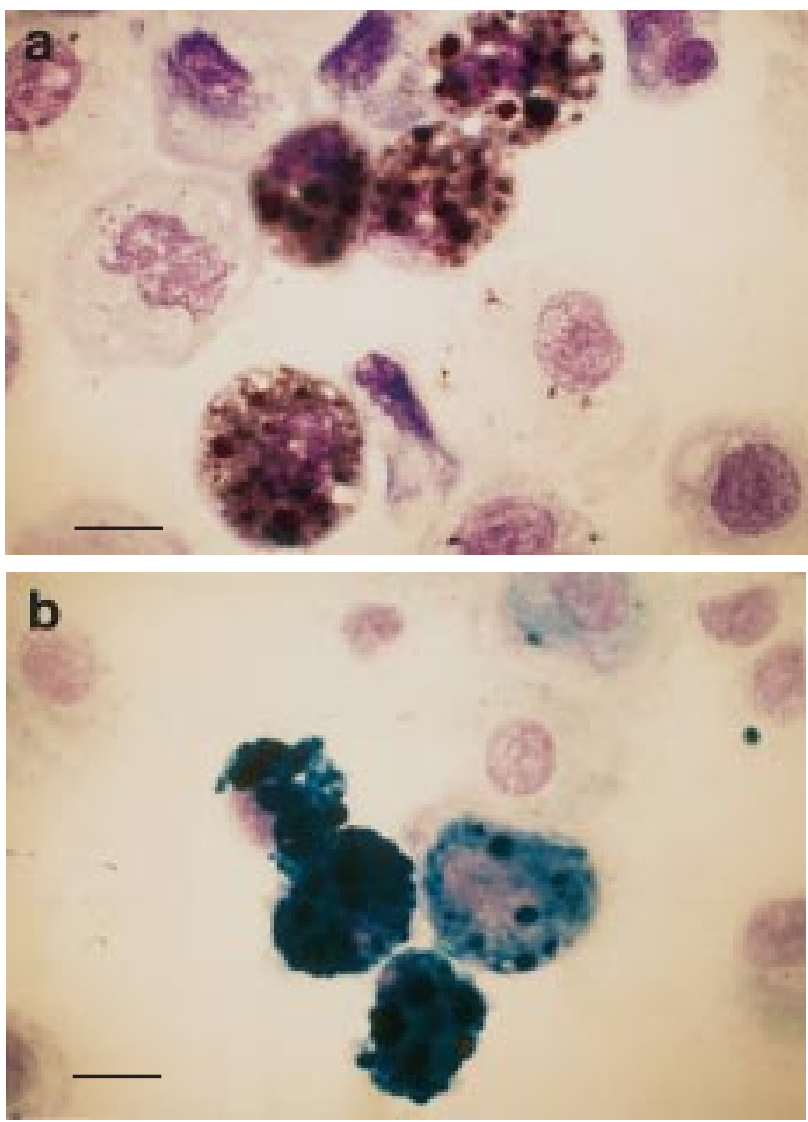

Fig. 1. - Cytospin preparation of bronchoalveolar lavage fluid cells from a vasculitis patient. a) The macrophages contain brownish bulky material on May-Grünwald Giemsa staining, which was further analysed by staining for iron. b) After Prussian blue staining, particles in the macrophages stain intensely blue, marking them as iron-containing material. (Internal scale bars $=10 \mu \mathrm{m}$.)

the score of GoLDE et al. [20] for the quantitation of haemosiderin-laden alveolar macrophages, which includes a grading of positive cells on a scale of 1-4 [20]. DE LASSENCE et al. [4] have demonstrated that the information conferred by this scoring system does not exceed that of the simple enumeration of iron-positive cells; for this reason the latter method was employed. The myeloperoxidase (MPO) activity in the BAL fluid was measured using the K-blue assay, as described by SEgELmark et al. [21], with minor modifications.

\section{Lung function testing and high-resolution computed tom- ography}

Pulmonary function, including static and dynamic volumes and single breath carbon monoxide diffusion capacity, was measured using a MasterLab unit (Jaeger, Würzburg, Germany) according to the criteria of the American Thoracic Society [22]. Values for the CO diffusion capacity were corrected for haemoglobin concentration and alveolar volume, as measured by the helium-dilution technique [23]. Pulmonary HRCT was performed using a Somatom Plus-S device (Siemens, Erlangen, Germany). Thin section radiographs (1 mm section thickness) were taken at maximum inspiration from the apex to the base of the 
lungs at $10-\mathrm{mm}$ intervals. Scans were reconstructed by means of a high spatial frequency reconstruction algorithm.

\section{Statistics}

Patient characteristics, BAL findings and the percentage of iron-positive alveolar macrophages are presented as medians and 25th and 75th percentiles of the median. Differences in these values between groups were tested for statistical significance using the Mann-Whitney test for independent samples. Differences in categorical values were tested for statistical significance using the Chisquared test or, in the event of small numbers, Fisher's exact test.

\section{Results}

A wide variation within and between disease groups was found in the number of iron-positive macrophages and a line of separation could be drawn between ANCA-associated vasculitis on the one hand and CVDs and RA on the other (fig. 2). In the former group, the majority of patients had iron-positive macrophages, with individual counts ranging up to $95 \%$ of total macrophages. By contrast, iron-positive macrophages were found in only 12 of the 44 patients with CVDs and RA. The highest individual count in these patients was $5 \%$, the median percentage was 0 and the $95 \%$ confidence interval $0-5 \%$. For further analyses, the latter value was taken to represent the upper limit of normal.

In the vasculitis group 26 of the $49(53 \%)$ patients had $>5 \%$ iron-positive macrophages. In accordance with the inclusion criteria, none of these patients had current or recent clinical or bronchoscopic evidence of pulmonary bleeding; they were thus diagnosed as having subclinical alveolar bleeding. To examine whether this finding correlated with indices of local or systemic disease activity, the 26 patients with elevated iron-positive cells were compared with the 23 vasculitis patients without this finding. In 20 of the former patients and 18 of the latter, the pulmonary HRCT results were abnormal (table 3 ). No statistically significant difference between the two subgroups was found with regard to the frequency of septal or nonseptal

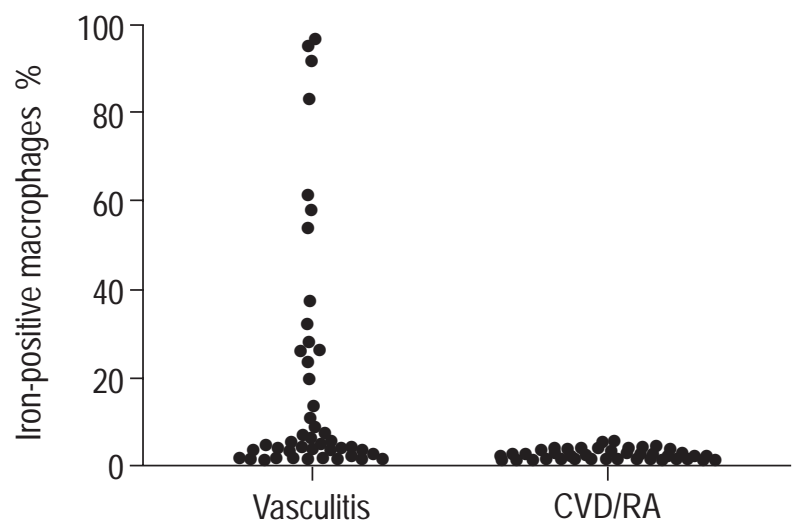

Fig. 2. - Percentage of iron-positive alveolar macrophages in vasculitis and collagen vascular diseases/rheumatoid arthritis (CVD/RA).
Table 3. - Abnormal high-resolution computed tomography (HRCT) findings of the patients wth antineutrophil cytoplasm antibody-associated vasculitis with high and low levels of iron-positive alveolar macrophages (AM) in bronchoalveolar lavage fluid

\begin{tabular}{lccc}
\hline & $\begin{array}{c}\text { High Fe+ } \\
\text { AM }\end{array}$ & $\begin{array}{c}\text { Low Fe+ } \\
\text { AM }\end{array}$ & p-value \\
\hline Low-attenuation opacities & 7 & 2 & NS \\
Septal/nonseptal lines & 11 & 10 & NS \\
Nodules & 8 & 7 & NS \\
Masses & 6 & 3 & NS \\
Consolidation & 1 & 3 & NS \\
\hline
\end{tabular}

Data are presented as number of patients with abnormal HRCT findings.

lines, nodules, masses, consolidations or low-attenuation opacities, although low-attenuation opacities were somewhat more common in the subgroup with elevated ironpositive cell counts (seven versus two patients). The two subgroups had an equivalent number of patients with an abnormal BAL cell profile (14 versus 14 patients) and lymphocyte elevation was the most common finding in both groups (table 4). However, the MPO activity in the BAL fluid, which is purported to reflect mainly local inflammatory activity, was significantly higher in the former than in the latter patients $(p=0.044)$ (table 4).

With respect to indices of systemic disease activity, the mean Disease Extent Index score was 6 (4-7.7) in the group with an elevated iron-positive cell count versus 4 (4-5) in the group without, a difference that is statistically significant $(\mathrm{p}=0.021)$ (table 1$)$. The frequency of upper respiratory tract, eye, nervous system, skin, heart and joint involvement was comparable in the two groups, but microhaematuria, suggestive of active kidney involvement, was present in 12 patients in the former and only two in the latter group ( $\mathrm{p}=0.005)$ (fig. 3). Moreover,

Table 4. - Pulmonary findings of the patients with antineutrophil cytoplasm antibody-associated vasculitis with high and low levels of iron-positive alveolar macrophages (AM) in bronchoalveolar lavage (BAL) fluid

\begin{tabular}{lccc}
\hline & $\begin{array}{c}\text { High Fe+ } \\
\text { AM }\end{array}$ & $\begin{array}{c}\text { Low Fe+ } \\
\text { AM }\end{array}$ & p-value \\
& $101(92-105)$ & $97(81-119)$ & NS \\
VC \% pred & $79(73-85)$ & $76(63-86)$ & NS \\
$\mathrm{FEV} / \mathrm{VC} \%$ & $94(82-106)$ & $104(82-107)$ & $\mathrm{NS}$ \\
$\mathrm{DL}, \mathrm{CO} \%$ pred & $87(77-103)$ & $93(83-99)$ & $\mathrm{NS}$ \\
$P \mathrm{a}, \mathrm{O}_{2} \mathrm{mmHg}$ & $37(35-40)$ & $36(34-38)$ & $\mathrm{NS}$ \\
$P \mathrm{a}, \mathrm{CO}_{2} \mathrm{mmHg}$ & & & \\
$\mathrm{BAL}$ cell profile & & & \\
Number of cells & $190(124-282)$ & $152(88-192)$ & $\mathrm{NS}$ \\
$10^{3} \cdot \mathrm{mL}^{-1}$ & & & \\
Lymphocytes \% & $15(9-29)$ & $22(8-41)$ & $\mathrm{NS}$ \\
Neutrophils \% & $1(0-2)$ & $1(0-2)$ & $\mathrm{NS}$ \\
Eosinophils \% & $0(0-0)$ & $0(0-0)$ & $\mathrm{NS}$ \\
Macrophages \% & $82(69-90)$ & $76(55-91)$ & $\mathrm{NS}$ \\
MPO in BAL fluid & $2.68(0.66-6.45)$ & $0.92(0-1.86)$ & 0.044 \\
U.mL ${ }^{-1}$ & & & \\
\hline
\end{tabular}

Data are presented as median (interquartile range). VC: vital capacity; FEV1: forced expiratory volume in one second; FEV1/ VC: Tiffeneau's index; $D \mathrm{~L}, \mathrm{CO}$ : diffusion capacity of the lung for carbon monoxide; $\mathrm{Pa}_{\mathrm{a}} \mathrm{O}_{2}$ : arterial oxygen tension; $\mathrm{Pa}_{\mathrm{a}} \mathrm{CO}_{2}$ : arterial carbon dioxide tension; MPO: myeloperoxidase; ns: not significant. $(1 \mathrm{mmHg}=0.133 \mathrm{kPa}$. $)$ 


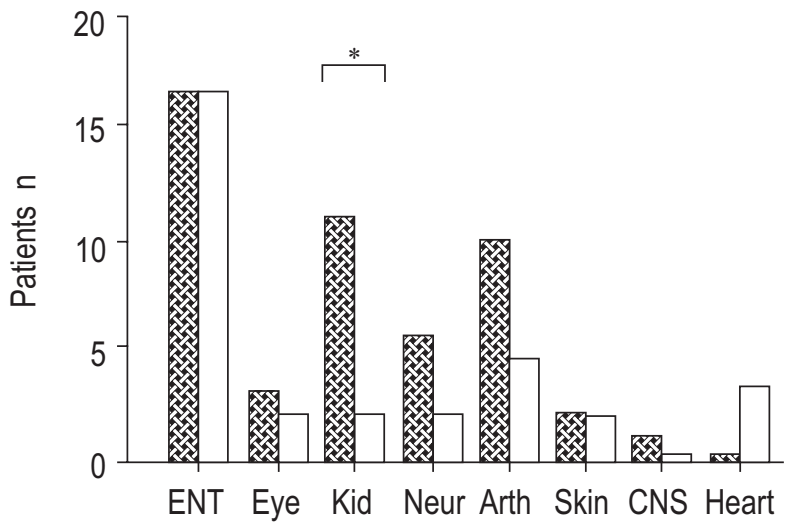

Fig. 3. - Extrapulmonary organ involvement in vasculitis patients with ( bronchoalveolar lavage fluid. ENT: ear/nose/throat; Kid: kidney; Neur: neuropathy; Arth: arthritis; CNS: central nervous system; *: $\mathrm{p}=0.005$.

the median ANCA titre was significantly higher in the patients with elevated numbers of iron-positive cells than in those without $(1: 256$ versus $1: 8, \mathrm{p}=0.030)$; however, the somewhat higher medians for erythrocyte sedimentation rate (ESR) and C-reactive protein concentration in the former did not differ significantly from those of the latter group. Collectively, therefore, patients with an elevated iron-positive cell count in their BAL fluid had more extended disease, a higher frequency of microhaematuria, a higher ANCA titre and a higher MPO activity in their BAL fluid than did patients without this finding, but no association was found between an elevated iron-positive cell count and acute phase reactant concentrations in the serum or the BAL cell profile.

A comparison of the groups with regard to treatment modalities provided no hint that treatment effects might be responsible for the differences in the number of ironpositive alveolar macrophages. An essentially equal percentage of patients in the two vasculitis subgroups received cyclophosphamide, cotrimoxazole or corticosteroid monotherapy. Furthermore, the CVD and RA patients treated with cyclophosphamide, azathioprine, methotrexate or corticosteroid monotherapy invariably had an iron-positive macrophage count $\leq 5 \%$, which further strengthened the supposition that elevated number of iron-positive cells are

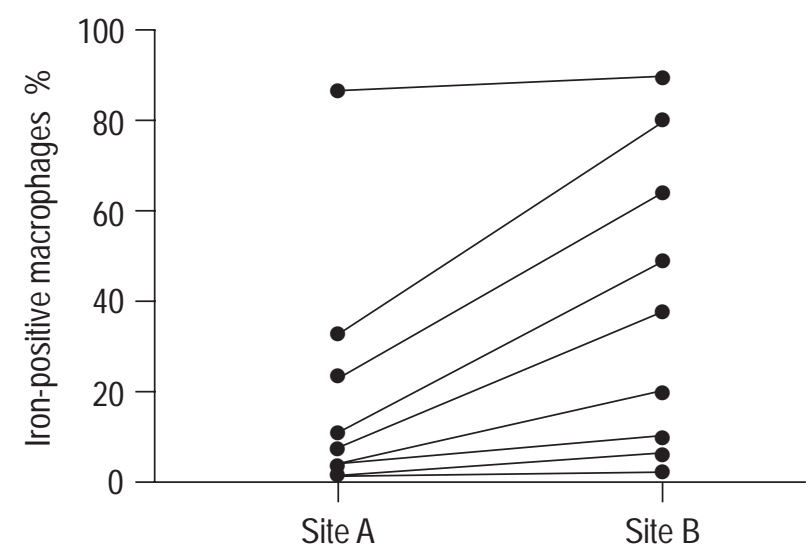

Fig. 4. - Percentage of iron-positive alveolar macrophages determined in two discrete lung segments. not causally related with any of these forms of treatment. The same conclusion was reached when this analysis was performed for low-dose heparin and low-dose acetylsalicylic acid.

To examine the spatial variability of the number of ironpositive macrophages, nine vasculitis patients underwent BAL of two segments in different lobes of the lung. In four patients, the percentage of iron-positive cells in the two segments differed by $<10$, whereas the values in the other five patients diverged widely (fig. 4).

\section{Discussion}

This study demonstrates that the presence of ironpositive alveolar macrophages is indeed a feature that distinguishes primary systemic vasculitis from CVDs and RA. Although the CVD and RA groups included a substantial number of patients with severe interstitial lung disease, only a minority of these patients had iron-positive macrophages, with individual counts of positive cells $\leq 5 \%$. The maximum value of $5 \%$ iron-positive cells in this group corresponds with figures reported for healthy subjects $[24,25]$. Conversely, 26 of the 49 vasculitis patients had $>5 \%$ iron-positive macrophages, and patients were seen who had inclusions of red cell fragments in $>90 \%$ of macrophages.

The presence of iron-positive cells in BAL fluid is by no means specific for any single condition, but can occur in diverse disorders [3]. To determine that this finding originated from microvascular disease in the present patients, a number of conditions had to be excluded during recruitment of the study groups. Firstly, patients with evidence of left ventricular failure or other conditions leading to elevated pulmonary capillary pressure were excluded. Since leakage of red cells into the alveoli can occur in haemorrhagic diatheses, all patients were screened for coagulation abnormalities and any abnormality resulted in exclusion from the study. In addition, patients with thrombocytopenia or uraemia, which can impair thrombocyte function, were excluded. Several patients had received prophylactic low-dose heparin or acetylsalicylic acid, but these patients were equally distributed between the vasculitis subgroups with high and low ironpositive cell counts. Also the finding that the CVD and RA patients receiving such prophylaxis had low ironpositive cell counts militates against an effect of these treatment modalities on the leakage of red cells into the alveoli. Since smoking can lead to increased numbers of iron-positive macrophages [4], only nonsmokers were admitted to this study. Reportedly, a number of bronchopulmonary infections can be associated with low-grade alveolar bleeding, a notorious example being pulmonary aspergillosis $[2,4]$. Such interference was ruled out by excluding patients with any evidence of pulmonary or systemic infection. Finally, a number of studies have reported alveolar bleeding in patients receiving high-dose polychemotherapy $[2,4,8]$. In addition to the aforementioned reasons for alveolar bleeding, combinations of which are present in many patients receiving polychemotherapy, the possibility that cytotoxic therapy alone can cause microvascular damage has been discussed but remains unresolved [8]. In the present study, no association was found between cyclophosphamide or any 
other treatment modality and an elevated number of ironpositive alveolar macrophages in the vasculitis patients, and none of the CVD or RA patients receiving cyclophosphamide had $>5 \%$ iron-positive cells. These findings do not support the idea that these treatment modalities may cause microvascular damage leading to alveolar bleeding.

This leads to the conclusion that the leakage of red cells into the alveoli in these patients was a result of microvascular incompetence due to the underlying vasculitis. Conceptually, this may be the pulmonary equivalent of the vascular pathology in the kidney which leads to haematuria. The most common form of renal involvement in ANCA-associated vasculitis is focal and segmental necrotizing glomerulonephritis [26]. The most common urine abnormality resulting from this is microhaematuria and, indeed, the presence of red cells of glomerular origin in the urine is a sensitive indicator of active glomerular disease. Furthermore, in the vast majority of cases, clinically manifest severe alveolar bleeding is associated with florid glomerulonephritis $[1,3,6]$, another indication that pulmonary and glomerular leakage may have a common pathogenetic basis. This possibility was pursued by comparing vasculitis patients with and without elevated iron-positive macrophages for indicators of local and systemic disease activity. Microhaematuria was in fact found to be significantly more common in patients with an elevated iron-positive cell count than in patients without. The former patients also had more extended disease, as reflected in their significantly higher Disease Extent Index scores and significantly higher ANCA titres.

As opposed to these indices of systemic disease activity, indices of pulmonary disease activity were only loosely related with the count of iron-positive macrophages. HRCT findings taken to suggest pulmonary involvement owing to the vasculitis were septal and nonseptal lines, nodules, masses, consolidations and low-attenuation opacities [27, 28]. Low-attenuation opacities suggestive of active inflammatory disease were somewhat more common in the patients with an elevated iron-positive cell count than in those without, although the difference fell short of statistical significance. Elevated numbers of lymphocytes, neutrophils or eosinophils or an elevated MPO activity in the BAL fluid were taken as measures of pulmonary disease activity [29]. While MPO activity was significantly higher in patients with an elevated iron-positive cell count than in patients without, this finding was unrelated to the BAL fluid cell profile. Collectively, an elevated iron-positive alveolar macrophage count was, thus, associated with selected indices of systemic and local disease activity, but this association was less than perfect and did not improve when this analysis was repeated with the cut-off point for the iron-positive cell count set at various levels $>5 \%$.

One probable reason for the association only being weak is probably the heterogeneous distribution of the underlying vascular pathology. Reportedly, a focal and irregular distribution of vasculitic, granulomatous, fresh and old tissue lesions is a characteristic feature of the pulmonary histopathology of ANCA-associated vasculitis [30, 31]. A focal distribution has also been described for parenchymal bleeding $[1,6,7]$. This can introduce a sampling error into BAL studies examining only single lung segments, as was verified in the present patients. Indeed, in the nine patients of the present study who underwent lavage of two segments in separate lobes of the lung, the values for iron-positive cells in the two segments differed substantially in five patients, whereas only in four patients did the percentage of iron-positive cells in the two segments differ by $<10 \%$. Thus, a large sampling error must be acknowledged, which could possibly be minimized in future by lavaging more than one segment.

Another potential reason for the weak association is the somewhat sluggish kinetics of uptake and degradation of red cells in alveolar macrophages. SHERMAN et al. [32] demonstrated that, after clinically manifest alveolar bleeding, it takes $\geq 50 \mathrm{~h}$ before haemosiderin-containing macrophages can be recovered from tracheal aspirates and $\geq 72 \mathrm{~h}$ before alveolar macrophages incubated in vitro with conditioned erythrocytes become positive for haemosiderin. They claimed that the clearance of haemosiderin-laden macrophages in vivo takes 2-4 weeks, but this estimate was based on repeat BAL examinations in only a small number of patients with alveolar bleeding. Furthermore, it is entirely unclear whether the dwell time of iron-positive macrophages in the lower respiratory tract is modified by disease or treatment-related effects. In acutely ill patients who are in a rapidly changing condition, the sluggish kinetics tend to temporally dissociate the iron-positive cell count from other clinical parameters and to disrupt correlations in cross-sectional analyses.

In conclusion, subclinical alveolar bleeding as indicated by haemosiderin-laden alveolar macrophages appears to be a common finding in pulmonary vasculitis. In the present cohort with active Wegener's granulomatosis or ChurgStrauss syndrome, an elevated number of such cells was found in $53 \%$ of the patients, whereas only very few such cells were present in patients with collagen vascular disease or rheumatoid arthritis, even in the presence of highly active inflammatory disease or advanced interstitial fibrosis. Pathophysiologically, this suggests different forms of vascular injury in primary systemic vasculitis as compared with collagen vascular disease and rheumatoid arthritis. Clinically, this can be a diagnostically helpful finding, in that the detection of high numbers of iron-positive macrophages favours the diagnosis of the former over the latter disorders. In patients with an established diagnosis of Wegener's granulomatosis or Churg-Strauss syndrome, a high iron-positive macrophage count tends to be associated with active disease, but single determination in just one segment appears to be fraught with a substantial sampling error. A longitudinal study is currently in progress to examine whether repeat determinations and samplings in more than one segment can enhance the diagnostic value of this finding in individual patients.

\section{References}

1. Travis WD, Colby T, Lombard C, Carpenter HA. A clinicopathologic study of 34 cases of diffuse pulmonary hemorrhage with lung biopsy confirmation. Am J Surg Pathol 1990; 14: 1112-1125.

2. Kahn FW, Jones JM, England DM. Diagnosis of pulmonary hemorrhage in the immunocompromised host. Am Rev Respir Dis 1987; 136: 155-160.

3. Green RJ, Ruoss SJ, Kraft SA, Bemy GJ, Raffin TA. Pulmonary capillaritis and alveolar hemorrhage - Update 
on diagnosis and management. Chest 1996; 110: 13051316.

4. de Lassence A, Fleury-Feith J, Escudier E, Beaune J, Bernaudin J-F, Cordonnier C. Alveolar hemorrhage. Diagnostic criteria and results in 194 immunocompromised hosts. Am J Respir Crit Care Med 1995; 151: 157-163.

5. Haworth SJ, Savage COS, Carr D, Hughes JMB, Rees AJ. Pulmonary haemorrhage complicating Wegener's granulomatosis and microscopic polyarteritis. $B M J 1985 ; 290$ : $1775-1778$.

6. Mark EJ, Ramirez JF. Pulmonary capillaritis and hemorrage in patients with systemic vasculitis. Arch Pathol Lab Med 1985; 109: 413-418.

7. Myers JL, Katzenstein A-L. Wegener's granulomatosis presenting with massive pulmonary hemorrhage and capillaritis. Am J Surg Pathol 1987; 11: 895-898.

8. Robbins RA, Linder J, Stahl MG, et al. Diffuse alveolar hemorrhage in autologous bone marrow transplant recipients. Am J Med 1989; 87: 511-518.

9. Leavitt LY, Fauci AS, Bloch DA, et al. The American College of Rheumatology 1990 criteria for the classification of Wegener's granulomatosis. Arthritis Rheum 1990; 33: 1101-1107.

10. Masi AT, Hunder GG, Lie JT, et al. The American College of Rheumatology 1990 criteria for the classification of Churg-Strauss syndrome (allergic granulomatosis and angiitis). Arthritis Rheum 1990; 33: 1094-1100.

11. Jennette JC, Falk R, Andrassy K, et al. Nomenclature of systemic vasculitides. Proposal of an international conference. Arthritis Rheum 1994; 37: 187-192.

12. Reinhold-Keller E, Kekow J, Schnabel A, et al. Influence of disease manifestation and antineutrophil cytoplasmic antibody titer on the response to pulse cyclophosphamide therapy in Wegener's granulomatosis. Arthritis Rheum 1994; 37: 187-192.

13. Nölle B, Specks U, Lüdemann J, Rohrbach MS, DeRemee RA, Gross WL. Anticytoplasmic autoantibodies: their immunodiagnostic value in Wegener's granulomatosis. Ann Int Med 1989; 111: 28-40.

14. Austin JHM, Müller NL, Friedman PJ, et al. Glossary of terms of the lungs: recommendations of the nomenclature committee of the Fleischer Society. Radiology 1996; 200: 327-331.

15. Tan AM, Cohen AS, Fries JF, et al. The 1982 revised criteria for the classification of systemic lupus erythematosus. Arthritis Rheum 1982; 25: 1271-1277.

16. Bohan A, Peter JB. Polymyositis and dermatomyositis. $N$ Engl J Med 1975; 292: 344-347.

17. Masi AT, Rodnan GP, Medsger TA, et al. Preliminary criteria for the classiification of systemic sclerosis (scleroderma). Arthritis Rheum 1980; 23: 581-590.

18. Arnett FC, Edworthy SM, Bloch DA, et al. The American
Rheumatism Association 1987 revised criteria for the classification of rheumatoid arthritis. Arthritis Rheum 1988; 31: 315-324.

19. Cartwright G. Diagnostic laboratory hematology. New York, Grune and Stratton, 1972; pp. 161-165.

20. Golde DW, Drew WL, Klein HZ, Finley TN, Cline MJ. Occult pulmonary haemorrhage in leukaemia. $B M J 1975$; 2: $166-168$.

21. Segelmark M, Persson B, Hellmark T, Wieslander J. Binding and inhibition of myeloperoxidase (MPO): a major function of ceruloplasmin? Clin Exp Immunol 1997; 108: 167-174.

22. American Thoracic Society. Standardization of Spirometry - 1987 Update: statement of the American Thoracic Society. Am Rev Respir Dis 1987; 136: 1285-1298.

23. American Thoracic Society. Single breath carbon monoxide diffusing capacity (transfer factor): recommendations for a standard technique. Am Rev Respir Dis 1987; 136: 1299-1307.

24. Linder J, Robbins RA, Rennard SI. Cytologic criteria for diffuse alveolar hemorrhage. Acta Cytol 1988; 32: 763.

25. Pérez-Arellano JL, Garcia J-EL, Macias MCG, Gomez FG, Lopez AJ, de Castro S. Hemosiderin-laden macrophages in bronchoalveolar lavage fluid. Acta Cytol 1992; 36: $26-30$.

26. Hoffman GS, Kerr GS, Leavitt RY, et al. Wegener's Granulomatosis. An analysis of 158 patients. Ann Intern Med 1992; 116: 488-498.

27. Maskell GF, Lockwood CM, Flower CDR. Computed tomography of the lung in Wegener's granulomatosis. Clin Radiol 1991; 48: 377-380.

28. Reuter M, Schnabel A, Wesner F, et al. Pulmonary Wegener's granulomatosis: correlation between highresolution CT findings and clinical scoring of disease activity. Chest 1998; 114: 500-506.

29. Cantin AM, North SL, Fells G, Crystal RG. Oxidantmediated epithelial cell injury in idiopathic pulmonary fibrosis. J Clin Invest 1987; 79: 1665-1673.

30. Travis WD, Hoffman GS, Leavitt RY, Pass HI, Fauci AS. Surgical pathology of the lung in Wegener's granulomatosis. Review of 87 open lung biopsies from 67 patients. Am J Surg Pathol 1991; 15: 315-333.

31. Gaudin PB, Askin FB, Falk RJ, Jennette JC. The pathologic spectrum of pulmonary lesions in patients with anti-neutrophil cytoplasmic autoantibodies specific for proteinase 3 and anti-myeloperoxidase. Am J Clin Pathol 1995; 104: 7-16.

32. Sherman JM, Winnie G, Thomasson MJ, Abdul-Karim FW, Boat TF. Time course of hemosiderin production and clearance by human pulmonary macrophages. Chest 1984; 86: 409-411. 